\title{
Background Subtraction for Effective Object Detection and its Parametric Evaluation
}

\author{
Siddhartha A. Meshram, Mr. Vishal Raskar \\ Department of Electronics \& Tele-communication Engineering \\ *M.E Scholar,M. E Teacher, Imperial College Of Engineering and Research, Pune(M.H)India
}

\begin{abstract}
Background subtraction is a powerful mechanism for detecting change in a sequence of images that finds many applications. The background subtraction methods apply probabilistic models to background intensities evolving in time nonparametric and mixture-of Gaussians. The main difficulty in designing a robust background subtraction algorithm is the selection of a detection threshold. In this we adapt threshold to varying video statistics by means of two statistical models. In addition to a nonparametric background model we introduce a foreground model based on small spatial neighborhood to improve discrimination sensitivity we also apply a Markov model to change labels to improve spatial coherence of the detections, the proposed methodology is applicable to other background models as well. The strength of the scheme lies in its simplicity and the fact that it defines an intensity range for each pixel location in the background to accommodate illumination variation as well as motion in the background. The efficacy of the scheme is shown through comparative analysis with competitive methods. Both visual as well as quantitative measures show an improved performance and the scheme has a strong potential for applications in real time surveillance.
\end{abstract}

Keyword: Background subtraction, tracking, object detection, surveillance.

\section{I] INTRODUCTION}

Object detection and tracking in video is a challenging background model. The desired effect of moving object problem and it has been extensively investigated in the extraction with a self-adaptive background model is past two decades and it has applications in numerous established in which self-adaptive updates according with fields, such as video compression, video surveillance, the changes in the scene. The moving objects detection human-computer interaction, Object detection and object system needs to deal with the following important tracking are two closely related processes. The former situations sudden and gradual changes in the light involves locating object in the frames of a video sequence conditions shadow region small movements of non-static while the latter represents the process of monitoring the objects such as waving tree and ocean waves; ghost. Then object's spatial and temporal changes in each frame. most important tasks in all such applications is change Object detection can be performed through various detection i.e. automatic segmentation of a video sequence approaches, such as region-based image segmentation, into static and changed (e.g. moving) areas. The numerous temporal differencing, active contour models and algorithms developed to date, the simplest ones are based generalized Hough transforms. In surveillance system on thresholding intensity differences. Since the detection video sequences are generally obtained through static results are sensitive to threshold selection (false positives cameras and fixed background.

versus misses) various threshold adaptation methods have

The background subtraction is an important first step for been proposed.

many vision problems and it separates objects from The model parameters have been estimated from background clutter usually by comparing motion patterns previous frames, and the detection process involves and facilitates subsequent higher-level operations such as thresholding the resulting PDF to thresholding tracking object identification cause the environment can probabilities instead of intensities and the approach is change substantially both in the short term and throughout more robust more constitutes a powerful tool for change the lifetime of the vision system background subtraction detection. In parallel change detection methods that were algorithms are expected to be robust. This is not always developed based on the maximum a posteriori probability easy to guarantee and many methods have been proposed (MAP) criterion. While some methods were formulated in in the literature. A popular approach called background discrete domain and used MRFs as prior models other subtraction is used in this scenario, where moving objects methods used variation formulations in continuous domain in a scene can be obtained by comparing each frame of the only embodying the spirit of the MAP criterion. The video with a background. In most of the suggested change detection performs well, it is computationally schemes the object detected is accompanied with complex; approximate, but faster, solution methods were misclassified foreground objects due to illumination developed. Then revisit background subtraction from the variation or motion in the background. hypothesis testing point of view and make two

In particular the background subtraction method is the contributions. Assuming spatial ergodicity we augment the most commonly used motion detection method and background model with an explicit foreground model and updating the background model are the keys to the ideal estimate its parameters from a small spatial neighborhood. 
To capture background dynamics, these approaches lack the two most compelling (and dynamic) aspects of the SG method: (1) the ability to account for transitory events, due to motion of foreground objects; and (2) simple model management. The absence of a hidden discrete state variable, the dynamic texture will slowly interpolate through all these states both the transition from occluded to turbulent, and turbulent to normal waves, will generate outliers which are incorrectly marked as foreground. Object detection in surveillance system background modeling plays a vital role and Wren et al. have proposed to model the background independently at each pixel acti location which is based on computation of Gaussian
probability density function (pdf) on the previous pixel values.

The Stauffer and Grimson developed a complex procedure to accommodate permanent changes in the background scene. In this each pixel is modeled separately by a mixture of three to five Gaussians. It uses three values to represent each pixel in the background image namely und the minimum intensity, the maximum intensity and In Marcenaro et al.'s study for relieving the effect of difference between consecutive frames of the training dynamic occlusion a multi-mode method to improve sequence. Jacques et al. brought a small improvement to accuracy and efficiency for tracking multiple targets in a the W4 model together with the incorporation of a crowed scene.

technique for shadow detection and removal [5]. McHugh A multi-class statistical model for the tracked objects but et al. proposed an adaptive thresholding technique by the background model is a single Gaussian per pixel. The means of two statistical models. One of them is attempt to mediate the effect of shadows appears to be nonparametric background model and the other one is somewhat successful but it is not clear behavior of their foreground model based on spatial information. In ViBe system would exhibit for pixels which did not contain each pixel in the background can take values from its these three distributions. For example pixels may present a preceding frames in same location or its neighbor, then it single background color or multiple background colors compares this set to the current pixel value in order to resulting from repetitive motions, shadows, or reflectance. determine whether that pixel belongs to the background The subtraction regions of interest are identified from the and adapts the model by choosing randomly which value foreground image based on the completeness of region to substitute from the background model. Then scheme contours. Additionally, detecting character regions from adopts a clustering-based feature, called fuzzy color the interested ones is then considered under the physical histogram $(\mathrm{FCH})$, which has an ability of greatly constraints of human body including the dynamic range of attenuating color variations generated by background pixel gray-level values and the shapes of regions. As motions while highlighting moving objects.

objects conforming to the constraints ellipses having the

Then scheme passes the texture information of each minimized areas that can cover the regions are constructed block through three cascading classifiers to classify them to derive their corresponding position parameters, ellipse as background or foreground. The results are then radiuses, and centroids. In the modified overlap tracker, integrated with a probabilistic voting scheme at pixel level four tracking states comprising new target, leaving target, for the final segmentation. Generally, shadow removal merged target and split target are used to understand algorithms are employed after object detection. Salvador et characters in the current frame. New target means an al. developed a three step hypothesis based procedure to object entering a video scenes.

segment the shadows. It assumes that shadow reduces the intensities followed by a complex hypothesis using the geometrical properties of shadows. It confirms the validity of the previous assumption. Choi et al. in their work of distinguished shadows from moving objects by cascading foreg three estimators, chromaticity, brightness, and local model of the background in the field of view of a camera is intensity ratio. A novel method for shadow removal using created and periodically updated for example to account Markov random fields (MRF) where shadow model is for illumination changes. In foreground detection a constructed in a hierarchical manner. It is observed that decision is made as to whether a new intensity fits the most of the simple schemes are ineffective on videos with background model the resulting change label field is fed illumination variations, motion in background, and back into background modeling so that no foreground dynamically textured indoor and outdoor environment etc. intensities contaminate the background model. Let I be a On the other hand, such videos are well handled by grayscale image sampled on 2-D lattice n€A€ $R^{2}$ extension complex schemes with higher computational cost.

to color images is straightforward. We denote a sequence 
of such images with $\mathrm{I}(\mathrm{n})$. We consider a nonparametric background model for its simplicity and performance.

$$
P_{B}\left(I^{K}(\mathrm{n})\right)=\frac{1}{N} \sum_{i-1}^{N} K\left(I^{k}(n)-I^{k-1}(n)\right)
$$

At each background location $\mathrm{n}$ of frame $\mathrm{k}$, the model uses intensity from recent frames to estimate background $\mathrm{PDF}$, where $\mathrm{K}$ is a zero-mean Gaussian with variance that, for simplicity, we consider constant throughout the sequence. If a similar foreground model is available then change labels can be estimated by evaluating intensity in a new frame against these two models.

$$
\frac{P_{B} I(n)}{P_{F} I(n)}=\prod \frac{\pi_{F}}{\pi_{B}}
$$

This entails testing labels (background) and (foreground) at each pixel of the current image by means of a binary hypothesis test (from now on the superscript is omitted to simplify notation) where on left are the probabilities I(n) of observing given it is the projection of either the background scene or a foreground object. On the righthand side, are the prior probabilities of observing background $\pi_{B}$ or foreground $\pi_{F}$, and a cost term? The ratio biases the decision based on the priori probabilities, the cost term accounts for unequal penalties assigned to the four decision/truth

$P_{F} I(n)=\frac{1}{N_{f}(n)} \sum_{m \in N_{f}} K(I(n)-I(m))$ scenarios.

Without an explicit foreground model, $P_{k}$ is usually considered uniform. Assuming fixed prior probabilities and collecting all constants in this leads to a fixedthreshold background test. A pixel is labeled as moving if its probability is sufficiently small and this simple test is prone to randomly-scattered false positives, even for low system. The additional post-processing has been proposed to correct such errors it is ad hoc and thus difficult to generalize. However this model is susceptible to illumination variation dynamic objects in the background and also to small changes in the background like waving of leaves etc. A number of solutions to such problems are reported where the background model is frequently updated at higher computational cost and thereby making them unsuitable for real time deployment.

\section{III] EXTRACTION OF FOUR GROUNDS}

In most background subtraction algorithms, $P_{F}$ it is assumed uniform thus preventing any decision bias by moving objects. An exception is the work of El Gammal et al. [3], who proposed foreground modeling for human body and of Sheikh and Shah, who proposed a general foreground model using past frames. While the first model is object-specific, the second one necessitates slow object motion as otherwise background samples contaminate $P_{F}$. Although this could be mitigated by object tracking such an approach would be illogical (track an object in order to detect it). Instead, we propose a foreground model based on small spatial neighborhood, i.e. in the same frame. Recently, we have demonstrated that periodicity in time also holds spatially; local-in-time and local-in-space models produce equivalent background characteristics.

$\mathrm{P}_{\mathrm{fi}} \mathrm{I}(\mathrm{n})=\frac{1}{N \mathrm{fi}_{\mathrm{i}}(\mathrm{n})} \sum_{\mathrm{m} \in \mathrm{Nf} \mathrm{f}_{\mathrm{i}(\mathrm{n})}} \mathrm{K}(\mathrm{I}(\mathrm{n})-\mathrm{I}(\mathrm{m}))$

Copyright to IJARCCE
After successfully developing the background model a local thresholding based background subtraction is used to find the foreground objects. This can be accomplished by modeling labels as a Markov random field of which is a particular realization. MRF models have been successfully used in motion detection reducing scattered false detections and smoothing region boundaries. We propose a Markov model within the binary hypothesis test while maintaining non parametric. Our approach extends early methods using single-Gaussian and uniform and shares Markovianity with more recent formulations. Also, despite the use of accelerated simulated annealing in, the computational complexity is high. Although one can seek local minima by means of one-at-a-time search such as the iterated conditional modes algorithm in this case a binary solution is identical to our binary hypothesis test. Also, our approach uses spatial periodicity whereas the one is based on temporal periodicity which necessitates slow motion or tracking of foreground objects.

$$
\frac{\mathrm{P}_{\mathrm{B}} \mathrm{I}(\mathrm{n})}{\mathrm{P}_{\mathrm{F}} \mathrm{I}(\mathrm{n})}=\prod_{\mathrm{P}\left(\mathrm{E}=\mathrm{e}^{\mathrm{F}}\right)}
$$

Since E there is a priori probabilities on the right-hand side are Gibbs distributions characterized by the natural temperature $\gamma$ cliques $\mathrm{C}$ and potential function $\mathrm{V}$ defined on. Choosing the cliques and potential function is crucial to the Gibbs model's effectiveness. We use 2 element cliques from the second-order, spatial Markov neighborhood (eight immediate neighbors), commonly used in image processing. An extension to higher neighborhood orders and more complex cliques is straightforward and increases spatial coherence of labels. The potential it should produce no penalty within a patch of identical label resulting in a high probability but it should penalize label fields with severe fragmentation. The potential is commonly used in such scenarios for its simplicity:

$$
\begin{aligned}
& \mathrm{V}(\mathrm{n}, \mathrm{m})=0, \text { if } \mathrm{e}(\mathrm{n})=\mathrm{e}(\mathrm{m}) \\
& 1, \text { if } \mathrm{e}(\mathrm{n}) \neq \mathrm{e}(\mathrm{m})
\end{aligned}
$$

Clearly, the inclusion of the foreground model improves the performance over the fixed-threshold approach and over the MRF approach although only slightly. For example for a given miss rate (FNR) the foreground-model approach results in a lower false alarm rate (FPR) than the fixed-threshold procedure, while for a given false alarm rate it produces a slightly lower miss rate.

$$
\frac{P_{B} I(n)}{P_{F} I(n)}=\Theta \exp _{\gamma}^{-1} Q_{F}(n)-Q_{B}(n)=v(n)
$$

Where $\mathrm{Q}_{\mathrm{F}}(\mathrm{n})$ four ground and $Q_{B}(n)$ background neighbours of $n$, an adjustment of in the fixed-threshold approach cannot accomplish the same error rates as the foreground-model approach. The inclusion of foreground model results in a clear decrease of misses and a slight increase of false alarms compared to the fixed-threshold method. However, most of the false alarms and misses are corrected by the addition of Markov model which significantly improves the detection 
performance. Again, although gains due to the foreground model which significantly improves the detection model are modest, it is clear that the miss rate within the performance. Again although gains due to the foreground "truck" object is reduced when compared with Markov- model are modest, it is clear that the miss rate within the only model shows results for video captured by network "truck" object is reduced when compared with Markovcamera. Then combined foreground-MRF model only bottom row shows results for video captured by significantly outperforms fixed thresholding as well as the network camera.

joint model and produces accurate results shows iterative

evolution of one object, shows the background probability The combined foreground-MRF model significantly as brightness level (top)). The complementarily of outperforms fixed thresholding as well as the joint model background and foreground probabilities (where is low, is $P_{B} / P_{F}$ and produces accurate results. The iterative high) leading to reinforced threshold adaptation, shows the evolution of one object shows the background probability corresponding evolution of the label field; since most gains as brightness level $P_{B}$ (top) followed by the evolution $P_{F i}$. occur in the first 2-3 iterations the process may be quickly terminated.

The complementarities of background and foreground

A similar convergence can be observed for the Markov probabilities leading to reinforced threshold adaptation. model, the window size (W) used during classification of a The corresponding evolution of the label field; since most pixel as stationary or non-stationary. A graphical variation gains occur in the first 2-3 iterations the process may be among these three parameters is for the "Lobby" video quickly terminated.

sequence, may be observed that for and the achieved maximum of $99.47 \%$. It may be observed that LIBS accurately detects objects in almost all cases with least misclassified objects. Then Moreover the shadows in "Intelligent Room" sequence are also removed by the proposed algorithm. Furthermore, object detection performance of LIBS scheme is superior to GMM and EGMM schemes; however it has similar performance with Reddy et al.'s scheme. But, LIBS scheme is computationally efficient compared to Reddy et al.'s scheme as the latter uses three cascading classifiers followed by a probabilistic voting scheme. A Markov model within the binary hypothesis test while probabilities.

maintaining non parametric. Our approach extends early methods using single-Gaussian and uniform and shares

\section{V] CONCLUSION}

In this we have tested the proposed models on other surveillance videos and confirmed the gains reported here. A Background subtraction based on these models currently serves as our main change detection algorithm in research on visual behavior analysis and classification. However the inclusion of a foreground model tends to grow the detected regions rather than shrink them. It is thus critical that the initial label field have as few false positives as possible. This can be accomplished via false discovery rate control that uses thresholding of significance scores instead of Markovianity with more recent formulations. In is a twoGaussian mixture and is uniform, we use more accurate nonparametric models. Also, despite the use of accelerated simulated annealing in, the computational complexity is high. Although one can seek local minima by means of one-at-a-time search, such as the iterated conditional modes algorithm, in this case a binary MAP-MRF solution is identical to our binary hypothesis test. Also, our approach uses spatial ergodicity whereas the one is based on temporal ergodicity which necessitates slow motion or tracking of foreground object.

1] T.Aach and A. Kaup "Bayesian algorithms for adaptive change detection in image sequences using Markov random fields," Signal Process, Image Commun., vol. 7, pp. 147-160, 1995.

[2] C. Stauffer and E. Grimson, "Learning patterns of activity using realtime tracking," IEEE Trans. Pattern Anal. Machine Intell., vol. 22, no. 8, pp. 747-757, 2000.

[3] A. Elgammal, R. Duraiswami, D. Harwood and L. Davis, "Background and foreground modeling using nonparametric kernel density for visual surveillance," Proc. IEEE, vol. 90, pp. 11511163, 2002.

[4] W. Huang, I. Gu and Q. Tian, "Statistical modeling of complex backgrounds for foreground object detection," IEEE Trans. Image Process., vol. 13, no. 11, pp. 1459-1472, Nov. 2004.

[5] N. Paragios and V. Ramesh, "A MRF-based approach to real-time subway monitoring," in Proc. IEEE Conf. Computer Vision Pattern Recognition, 2001, pp. 58-65.

\section{IV] PERFORMANCE ANALYSIS}

Clearly, the inclusion of the foreground model improves the performance over the fixed-threshold although only slightly in each case. For example for a given miss rate (FNR) the foreground-model approach results in a lower false alarm rate (FPR) than the fixed-threshold procedure while for a given false alarm rate it produces a slightly lower miss rate. Thus an adjustment of in the fixedthreshold approach cannot accomplish the same error rates as the foreground-model approach. For a given the inclusion of foreground model results in a clear decrease of misses and a slight increase of false alarms compared to the fixed-threshold method. However most of the false alarms and misses are corrected by the addition of Markov J.Migdal and E. L. Grimson, "Background subtraction using Markov thresholds," in Proc. IEEE Workshop Motion and Video Computing, WACV/MOTIONS'05, 2005, vol. 2, pp. 58-65.

[7] Y. Sheikh and M. Shah, "Bayesian modeling of dynamic scenes for object detection," IEEE Trans. Pattern Anal. Machine Intell., vol. 27, no. 11, pp. 1778-1792, 2005.

[8] P.-M. Jodoin, M. Mignotte and J. Konrad, "Statistical background subtraction using spatial cues," IEEE Trans. Circuits Syst. Video Technol., vol. 17, pp. 1758-1763, Dec. 2007.

[9] J. McHugh, "Probabilistic Methods for Adaptive Background Subtraction,” M.S. thesis, Boston Univ., Boston, MA, Jan. 2008.

[10] J. McHugh, J. Konrad, V. Saligrama, P.M. Jodoin and D. Castanon, "Motion detection with false discovery rate control," in Proc. IEEE Int. Conf. Image Processing. Oct. 2008. 